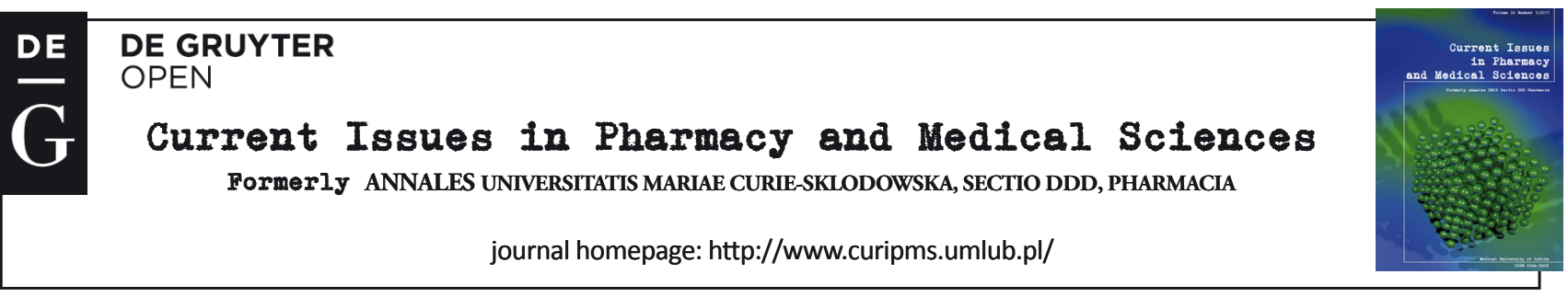

\title{
Advances in surgery for peritoneal surface malignancies
}

\author{
Jerzy Mielko ${ }^{1}$, Karol Rawicz-Pruszynski ${ }^{1}$, Tomasz Jastrzebski ${ }^{2}$, Wojciech P. Polkowski ${ }^{1 \star}$ \\ ${ }^{1}$ Department of Surgical Oncology, Medical University of Lublin, Staszica 11, 20-081 Lublin, Poland \\ 2 Department of Surgical Oncology, Medical University of Gdansk, Poland
}

\begin{tabular}{l} 
ARTICLE INFO \\
\hline Received 27 December 2017 \\
Accepted 29 December 2017 \\
\hline Keywords: \\
adenomatosis, \\
peritoneal surface \\
malignancies, \\
gastric cancer, \\
colorectal cancer, \\
cytoreductive surgery, \\
hyperthermic intraperitoneal \\
chemotherapy.
\end{tabular}

\begin{abstract}
Currently, patients with isolated peritoneal surface malignancies are treated with a combination of cytoreductive surgery (CRS) and hyperthermic intraperitoneal chemotherapy (HIPEC). This combination should now be considered the standard of care for appendiceal cancers (including pseudomyxoma), colorectal cancer and peritoneal mesothelioma, while patients with peritoneal metastases from ovarian or gastric cancer may be treated within clinical trials. At present, 8 clinical centres in Poland perform CRS and HIPEC. The unanswered problems of combined intraperitoneal therapy were an impulse for the organization of the $4^{\text {th }}$ International Conference "Advances in Surgical Oncology" that was held on November 23-24, 2017, in Lublin (Poland), and the Polish chapter of the Peritoneal Surface Oncology Group International (PSOGI) was established as a result of this meeting. This special issue of the Current Issues in Pharmacy and Medical Sciences is dedicated to the current therapeutic difficulties discussed during the two days of the conference - experiences that were exchanged to set in motion further directions for the improvement of intraperitoneal treatment.
\end{abstract}

\section{INTRODUCTION}

The peritoneal surface is commonly manifested in digestive and gynaecologic malignancies. At present, patients with isolated peritoneal surface malignancies are treated with a combination of cytoreductive surgery (CRS) and hyperthermic intraperitoneal chemotherapy (HIPEC). The combination should now be considered the standard of care for appendiceal cancers (including pseudomyxoma), colorectal cancer (CRC) and peritoneal mesothelioma, while patients with peritoneal metastases (PM) from ovarian or gastric cancer (GC) may be treated within clinical trials. A monography with current state of the art in this field was recently published for Polish readers (1).

At present, only selected patients with limited PM arising from CRC can be treated using CRS combined with HIPEC. Still, a recent review of 10 clinical trials that were reported during the $10^{\text {th }}$ International Congress on Peritoneal Surface Malignancies that met in November 2016, in Washington DC, revealed four trials (Dutch, French, Italian and Spanish) focused on a preventive strategy. Additionally, CRS and HIPEC for PM were compared in other trials with different intraperitoneal treatments without hyperthermia, or with no intraperitoneal treatment, or with intravenous chemotherapy (2). Hence, the number of recruiting trials evaluating

\footnotetext{
* Corresponding author

e-mail: wojciech.polkowski@umlub.pl
}

CRS and HIPEC in the prevention or therapy of CRC PM is increasing, and, probably, in the coming years, clinical results will change the current clinical practice.

Although there is a near universal standardization regarding CRS (peritonectomy), a standardization of various intraperitoneal chemotherapy drugs and modalities is still lacking. Pharmacologic evidence should, thus, be generated to answer important questions raised by the myriad of variables associated with intraperitoneal chemotherapy (3). Until standardised chemotherapy regimens are developed and generally accepted, current clinical practice will not be changed.

There is also no standard treatment for PM from GC. The published, as well as the registered ongoing clinical trials on CRS plus HIPEC for GC PM were recently critically evaluated (4). Accordingly, the natural course of GC with PM is less than 5 months, whereas (cytoreductive) gastrectomy prolongs overall survival (OS) up to 8 months. The median OS after CRS plus HIPEC was: in prospective studies -11 months; in case-control and in cohort studies - 13 months. Moreover, the median 1-, 2-, and 5-year survival rates after CRS plus HIPEC were $50 \%, 36 \%$ and $13 \%$, respectively. Furthermore, there is no statistically significant increase in serious adverse events that can be directly attributed to CRS plus HIPEC. The combination of CRS and HIPEC is, therefore, a promising integrated treatment strategy for GC PM 
that has encouraging initial results, calling for urgent further evaluation of this strategy in randomized control trials.

These unresolved problems of the CRS and HIPEC for peritoneal surface malignancies were an impulse for the organization of the $4^{\text {th }}$ International Conference "Advances in Surgical Oncology" that was held on November 23-24, 2017, in Lublin (Poland). The president of the Scientific Committee was professor Wojciech P. Polkowski, from the Chair of Oncology and the Department of Surgical Oncology of the Medical University of Lublin. The Honorary Patronage over the conference was taken by the Rector of the Medical University of Lublin, the Director of Clinical Hospital No. 1 in Lublin, the Lublin Medicine Cluster, and the Mayor of the city of Lublin (which this year celebrates the $700^{\text {th }}$ anniversary of obtaining city rights). The conference was endorsed by both, the Polish (PTChO) and European (ESSO) Societies of Surgical Oncology.

Foreign faculty came from France (Olivier Glehen, Lyon), Germany (Beate Rau, Berlin and Hubert Leebman, Regensburg), Italy (Daniele Marrelli, Siena), the Netherlands (Johan Offerhaus, Utrecht), Spain (Santiago GonzalezMoreno, President of the ESSO), and from Ukraine (Roman Yarema, Lviv). The following problems were topics for discussion: diagnostic imaging of peritoneal metastases, practical aspects of diagnostic laparoscopy, cytological and molecular assessment of peritoneal fluid, scientific evidence for CRS and HIPEC in the treatment of peritoneal surface cancer, comprehensive treatment of peritoneal metastases from gastric cancer, prophylactic (intraperitoneal) gastric cancer, metastases to the ovaries with tumours of the digestive system (Krukenberg tumours), as well as innovations and the future of intraperitoneal treatment.

During the conference, Professor G. Johan A. Offerhaus, Doctor Honoris Causa of the Medical University of Lublin, presented a special lecture entitled What molecular sub-types of gastric cancer have potential for peritoneal dissemination? In addition, Professor Olivier Glehen, chairman of RENAPE (French Network on rare peritoneal disease) and BIG-RENAPE (National Clinic-Biological Database on Digestive Peritoneal Carcinomatosis) presented the principles of preventive intraperitoneal therapy in patients with gastric cancer. Finally, the results of the combined treatment of locally advanced and disseminated patients with peritoneal gastric cancer were presented by doc. Roman Yarema, from the Lviv Medical University. The Polish chapter of the Peritoneal Surface Oncology Group International (PSOGI) was established as a result of this meeting.
About 100 participants from Poland and abroad took part in the conference. Specialists from many European centres thus had the opportunity to exchange experiences and to get acquainted with the Polish point of view and with current treatment techniques. At present, 8 clinical centres in Poland perform CRS and HIPEC. The first CRS and HIPEC in Poland was done in 1998, at the Department of Surgical Oncology of the Medical University of Gdansk, by Tomasz Jastrzebski. Since then, nearly 600 HIPEC treatments were undertaken in Poland, and the medical community has been able to verify the effectiveness of the method. In Lublin, the first HIPEC operation was performed in November 2010, by prof. Polkowski's team. The Department of Surgical Oncology of the Medical University of Lublin currently has the greatest experience in this technique in the country, with almost 200 CRS and HIPEC operations performed. Taking into account the experience of Polish surgeons in the treatment of peritoneal metastases, the organization of international conferences and domestic CRS and HIPEC workshops, the President of the ESSO has proposed the creation of a Centre of Excellence in Poland for this multidisciplinary training.

In the summary of the conference, prof. O. Glehen drew attention to the high substantive level of both the speakers' speeches and the held discussions on the addressed subjects. He also noted the excellent organization of the conference and the selection of topics that reflect the global nature of the currently discussed issues. The conference was also an opportunity to discuss new multi-institutional projects in which the participation of domestic and foreign centres is included.

This special issue of the Current Issues in Pharmacy and Medical Sciences is dedicated to the current therapeutic problems discussed during the two days of the conference. Such experiences will set forth further directions for the improvement of intraperitoneal treatment.

\section{REFERENCES}

1. Jeziorski A, Jastrzebski T, Polkowski WP. Biblioteka Chirurga Onkologa. Tom 9. Terapia CRS + HIPEC. Gdansk.: VM Media; 2017.

2. Pinto A, Eveno C, Pocard M. Update on clinical trials in colorectal cancer peritoneal metastasis. Int J Hyperthermia. 2017;33(5):543-7.

3. Lemoine L, Sugarbaker P, Van der Speeten K. Drugs, doses, and durations of intraperitoneal chemotherapy: standardising HIPEC and EPIC for colorectal, appendiceal, gastric, ovarian peritoneal surface malignancies and peritoneal mesothelioma. Int J Hyperthermia. 2017;33(5):582-92.

4. Ji ZH, Peng KW, Yu Y, Li XB, Yonemura Y, Liu Y, et al. Current status and future prospects of clinical trials on CRS + HIPEC for gastric cancer peritoneal metastases. Int J Hyperthermia. 2017;33(5):562-70. 